\title{
Die Kleinformen der Frostmusterböden: Vergleich Arktis - Alpen - Tropisches Hochgebirge
}

\author{
Ralf Freund \\ Geographisches Institut, Universität Zürich
}

\section{Einleitung}

Über die Frostmusterböden ist in der Literatur schon recht häufig berichtet worden, vorwiegend aber im kleinräumlichen Rahmen. Diese Bodenformen sind Erscheinungen des subnivalen Höhenbereiches (Troll, 1944; Furrer, 1953), desen oberen Abschluß die Schneegrenze bildet und dessen Untergrenze neuerdings Fitze (1969) dargestellt hat. Innerhalb dieser Höhenstufe erreicht die Solifluktion ihre größte Aktivität und markante Formausprägung. Unter Solifluktion verstehe ich wie schon Troll (1944) eine langsame Bewegung der obersten Bodenschicht unter dem Regime des Bodenfrostes, beeinflußt durch Vegetation und Geländefaktoren (Fitze, 1969). Solifluktionsformen sind daher prinzipiell in allen Frostklimaten der Erde zu erwarten.

Es war bisher nicht üblich, sich allein mit den Kleinformen der Solifluktion zu befassen. Da mir aber innerhalb eines Jahres die Möglichkeit geboten wurde, gleich an zwei Expeditionen in die Arktis (West-Spitzbergen) und ins tropische Hochgebirge (Kilimandjaro) teilzunehmen, lag es auf der Hand, sich vom Stoff her einzuschränken, um dafür einen großräumlichen Vergleich anzupeilen.

Zu den Kleinformen zähle ich:

Zellenböden
Steinpolygone (mit Riß)
Steinstreifen (mit Riß)
Erdknospen
Erdstreifen

Die beiden letztgenannten Bodenmuster werden auch oft als Miniaturformen bezeichnet (Furrer, 1965). Ich möchte den Ausdruck vermeiden, da die Feldarbeit in allen drei Gebieten zeigte, daß an sich einander entsprechende Formen oft bedeutende Unterschiede in den Ausmaßen aufweisen. Unter Kleinformen sollen die kleinsten Solifluktionserscheinungen bis zu einer Obergrenze von $20-30 \mathrm{~cm}$ (Formdurchmesser bzw. Streifenabstand) verstanden sein.

Uber Vorkommen von Kleinformen in aller Welt hat Troll (1944) in seinem ausführlichen Werk über die Solifluktion berichtet. Er spricht vom "tropischen Typ» und nennt an arktischen Kleinformen bloß die Zellenböden.Vor ihm hat Flückiger
(1934) die kleinen Frostmusterböden am Kilimandjaro beobachtet, während Mohaupt (1932) nach seinen Forschungen in den Alpen für eine Form den Begriff «Erdstreifen» geprägt hat. Von diversen neueren Arbeiten möchte ich Furrer (1954-1969) und Kelletat $(1969,1970)$ herausgreifen, die aber beide jeweils auch Großformen behandeln.

Aus dem Gesagten ergibt sich das Verlangen, über die Kleinformen der Frostmusterböden eine vergleichende Untersuchung möglichst weltweit - wenigstens über die drei Arbeitsgebiete hinweg - anzusetzen. Eine kleine Auswahl davon soll dieser Aufsatz vermitteln.

\section{Die Arbeitsgebiete}

Das Untersuchungsgebiet in Graubünden, in einem Gebirge der gemäßigten Breiten, umfaßt die Hochregionen (2200-3000 $\mathrm{m}$ ü. M.) in der Umgebung von Arosa und der Lenzerheide; die Grenzen werden durch den Rhein, die Albula, das Landwasser und die Landquart gebildet (LK $1: 50$ 000, Bl. 247, 248, 257, 258). Die für die subnivalen Bodenformen günstigen, ziemlich ebenen Flächen sind eher spärlich vertreten, denn die Massenerhebung ist nicht eben bedeutend, die Zertalung dagegen intensiv. Auf dem Parpaner Rothorn sind bereits Studien über die Kleinformen der Frostmusterböden gemacht worden (Freund, 1968), und zudem laufen seit etlichen Jahren Messungen der Bodentemperatur, die noch ausgewertet werden.

Die beiden Arbeitsgebiete im Ausland (Fig. 1) waren vorausbestimmt durch den Standort der beiden Expeditionen. Auf Spitzbergen (norweg. Svalbard) ist es die Umgebung der norwegischen Dauersiedlung Longyearbyen im Westteil der arktischen Hauptinsel mit einem reichen Angebot an ebenen und leicht geneigten Flächen und mit viel Schuttmaterial (0-1050 m ü. M.). Am Kilimandjaro liegen die Regionen mit Solifluktionsformen wegen des tropischen Klimas erwartungsgemäß höher als in den Alpen; die untersuchten Gebiete erstrecken sich von 3800 bis $5800 \mathrm{~m}$ ü. M., und das weite, größtenteils sehr flache Gelände zwischen Hauptund Nebengipfel (Kibo bzw. Mawenzi) ist vollständig übersät mit feinem Schuttmaterial. 


\section{Der Kleinformenschatz}

An Kleinformen sind in den drei klimatisch stark voneinander verschiedenen Untersuchungsgebieten alle Typen vertreten, allerdings nicht in gleichen Mengen. Es zeigt sich, daß den Erdstreifen (geneigte Flächen) die größte Bedeutung zukommt. Sie besitzen die charakteristischsten Merkmale und überwiegen mengen- und flächenmäßig klar über alle anderen Kleinformen. Die Erdknospen sind als homologe Bildungen auf ebenen Flächen zu betrachten.

\subsection{Erdknospen}

Abbildung 1 zeigt ein gut aussortiertes Beispiel dieses Frostbodentyps. Die Entstehung der Erdknospen scheint heute gesichert, weil offenbar die Feinarde die oberflächliche Schuttdecke mit Hilfe des Bodenfrostes von unten her punktförmig durchstößt. Alle untersuchten Formen von Spitzbergen, Graubünden und vom Kilimandjaro besitzen als gemeinsame Merkmale:

aufgewölbte Feinerde mit Blasen im Innern;

Schutt in flachen Mulden ohne Riß;

sog. Rahmenauskleidung (feines Kiesmaterial zwischen Grobschutt des Rahmens und Feinerde im Zentrum).
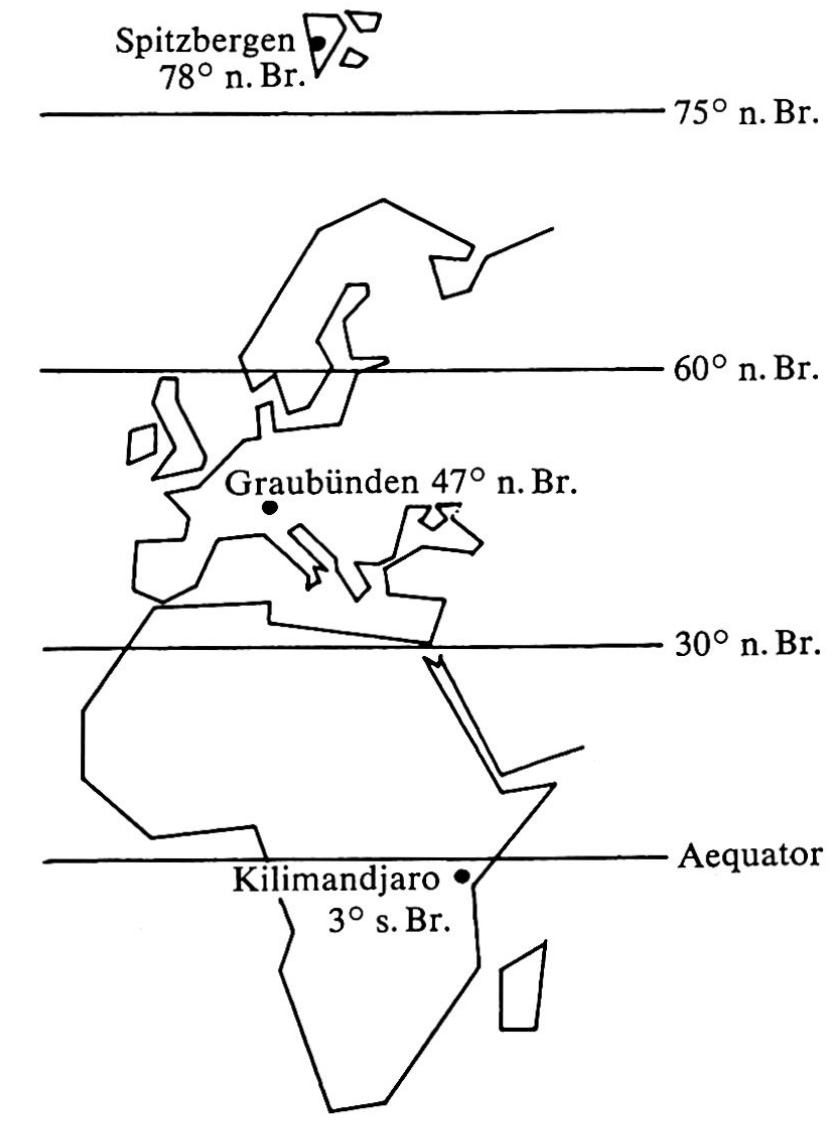

Figur 1. Die Lage der drei Arbeitsgebiete

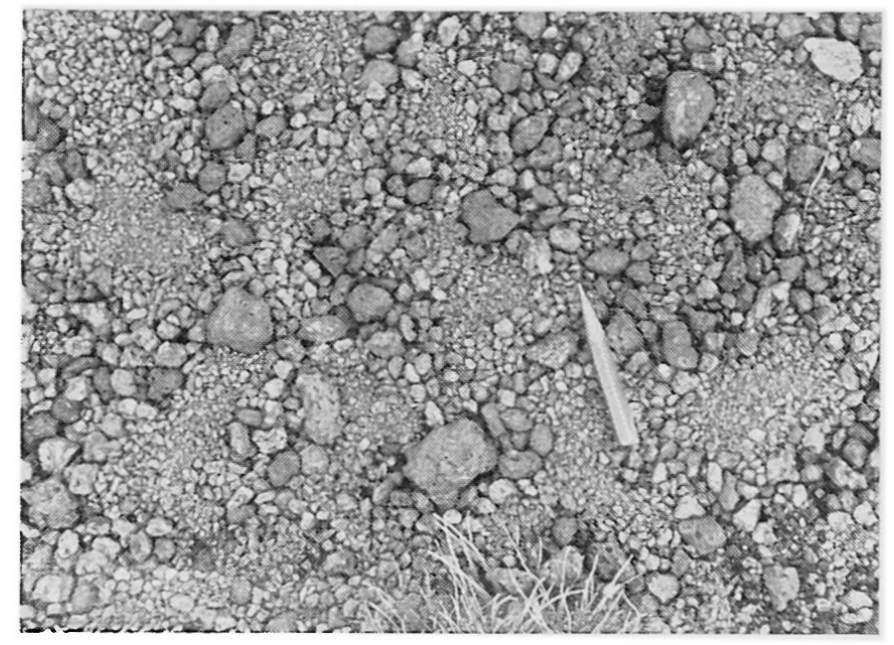

Abbildung 1. Erdknospen am Kilimandjaro, $4330 \mathrm{~m}$ ü. M., Bleistift $5 \mathrm{~cm}$

Weitere Merkmale, besonders die Größenverhältnisse, sind im folgenden tabellarisch zusammengestellt:

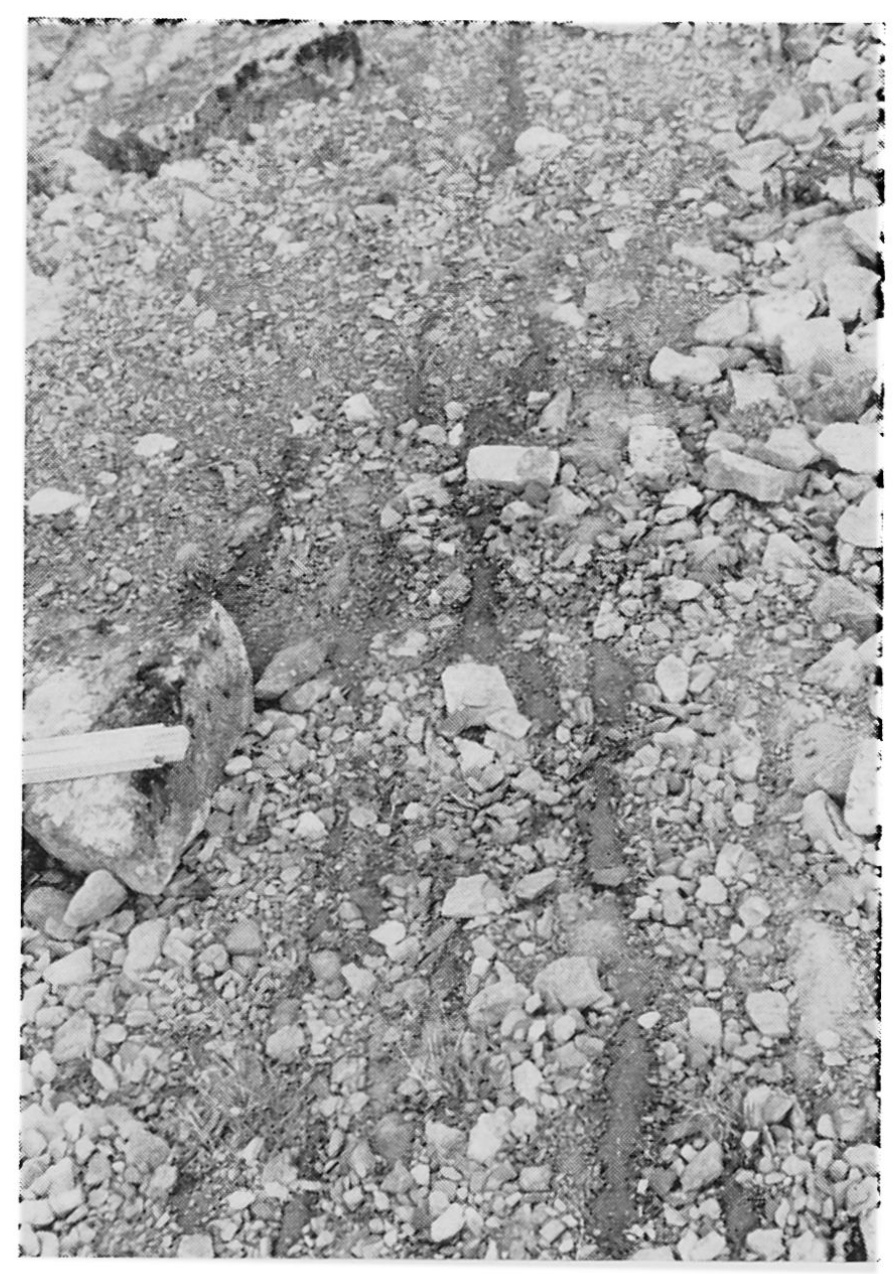

Abbildung 2. Erdstreifen auf West-Spitzbergen. Hotellneset, $130 \mathrm{~m}$ ü. M., $29^{\circ}$ E. Klappmeter $20 \mathrm{~cm}$ 


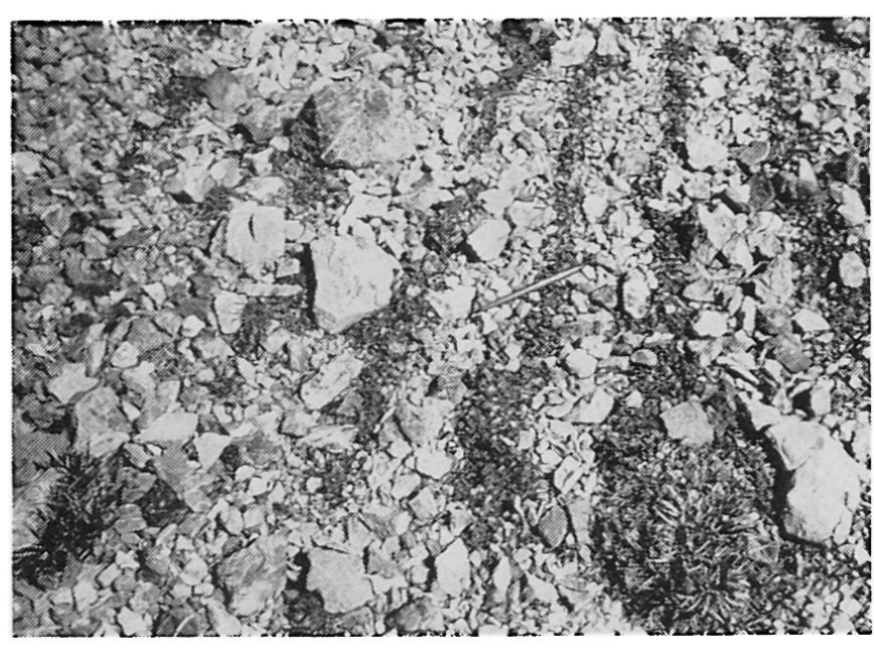

Abbildung 3. Erdstreifen in Mittelbünden. Maienfelder Furgga, $2440 \mathrm{~m}$ ü. M., $8^{\circ} \mathrm{S}$. Kugelschreiber $13 \mathrm{~cm}$

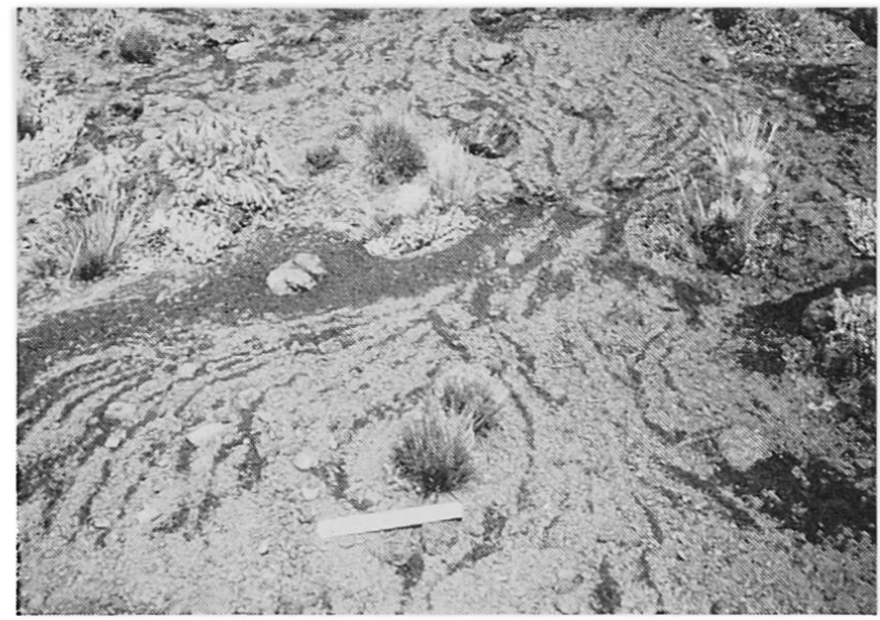

Abbildung 4. Erdstreifen am Kilimandjaro. Sattel, $4330 \mathrm{~m}$ ü. M., $5^{\circ} \mathrm{S}$. Klappmeter $20 \mathrm{~cm}$

dieselben wie bei den Erdknospen - waren vorhanden (Abb. 2). Nach Furrer (1969) können aber im Ostteil der Inselgruppe die Erdstreifen auch große Areale bedecken.

$\phi$ der Form

Rahmenbreite

Rahmentiefe

Aufwölbung

Verbreitung

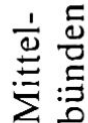

$5-15 \mathrm{~cm}$

3- $6 \mathrm{~cm}$

$2-5 \mathrm{~cm}$

$1-2 \mathrm{~cm}$

0-1050 $\mathrm{m}$ ü. $\mathrm{M}$.
$8-15 \mathrm{~cm}$

$3-10 \mathrm{~cm}$

$2-5 \mathrm{~cm}$

$1-3 \mathrm{~cm}$

$2350-2800-38$ m ü. M. m ü. $M$.

\subsection{Erdstreifen}

Was sich für die Erdknospen ergeben hat - nämlich ein größenmäßiges Entsprechen der arktischen und der «gemäßigten» Form gegenüber der auffallend kleineren tropischen Form -, zeigt sich noch deutlicher bei den Erdstreifen. Dazu kommt, daß die winzigen Streifenmuster am Kilimandjaro gewissermaßen als Ausgleich viele hundert Meter Länge erreichen können.

Auf West-Spitzbergen habe ich nur wenige und leider keine flächenhaften Funde machen können, aber die typischen Merkmale von Erdstreifen -

Im Raume Mittelbünden sind diese Streifenmuster in der Subnivalstufe häufig und in allen Gesteinen anzutreffen. Nach eigenen Beobachtungen wird die Materialsortierung im Dolomitschuitt am besten ausgebildet (Abb. 3). Zu den anderen Merkmalen tritt der Stromlinienverlauf, das heißt das stromlinienartige Umfließen eines Hindernisses (großer Stein, Vegetationspolster), wie das bereits Mohaupt (1932) beschrieben hat.

Am Kilimandjaro scheinen die Erdstreifen «zu Hause" zu sein, sind sie doch praktisch auf Schritt und Tritt und oft über unabsehbare Flächen hinweg zu finden. Hier zeigt es sich, daß das bisher geforderte Merkmal der strengen Parallelität der Streifen nicht durchwegs vorhanden ist; auch Verzweigungen und Vereinigungen von Feinerdestreifen sind wiederholt zu beobachten. Das Prinzip des Stromlinienverlaufes ist wunderschön ausgebildet (Abb. 4).

Wieder soll ein Zahlenvergleich in Tabellenform folgen:

$\begin{array}{lll}\text { Spitzbergen } & \text { Mittelbünden } & \text { Kilimandjaro } \\ 4-6 \mathrm{~cm} & 5-10 \mathrm{~cm} & 2-4 \mathrm{~cm} \\ 4-6 \mathrm{~cm} & 5-10 \mathrm{~cm} & 1-3 \mathrm{~cm} \\ \text { bis } 6 \mathrm{~m} & \text { bis } 15 \mathrm{~m} & \text { bis viele } 100 \mathrm{~m} \\ 3 \mathrm{~cm} & 3-5 \mathrm{~cm} & 1-3 \mathrm{~cm} \\ 1 \mathrm{~cm} & 1-3 \mathrm{~cm} & 0,2-1 \mathrm{~cm} \\ 5-29^{\circ} & 5-30^{\circ} & 4-35^{\circ} \\ 0-1050 \mathrm{~m} \text { ü. M. } & 2350-2800 \mathrm{~m} \text { ü. M. } & 3850-5600 \mathrm{~m} \mathrm{ü.} \mathrm{M.}\end{array}$




\subsection{Situmetrie und Granulometrie der Erdstreifen}

Bei den Einregelungsmessungen und deren graphischer Darstellung als Situgramm wurde nach der verfeinerten Methode von Bachmann (1966) vorgegangen. Figur 2 zeigt ein sogenanntes Sammelsitugramm, das wesentlich aussagekräftiger ist als ein Einzelsitugramm. Dabei werden die Mittelwerte aus vielen Einzelmessungen dargestellt.

Die Sammelsitugramme aus dem arktischen und tropischen Klimabereich sind beinahe identisch mit dem vorliegenden. Das graphische Bild sagt aus, daß die Steine mit ihrer Längsachse vorzüglich in der Fallinie des Hanges liegen, während der Anteil bis zu den querliegenden kontinuierlich abnimmt. Obwohl die Erdstreifen in den drei Untersuchungsgebieten verschiedene Dimensionen aufweisen, ergeben sich mit der Einregelung keine relevanten Unterschiede.

Komplizierter wird es bei den Korngrößenverhältnissen. Figur 3 zeigt Granulogramme von Erdstreifenproben aus West-Spitzbergen, Figur 4 solche von Proben vom Kilimandjaro. Die von mir untersuchten arktischen Erdstreifen besitzen einen relativ bedeutenden Anteil an Ton (kleinste Fraktion). Nach Semmel weist diese Tatsache auf ganz alte Böden hin. Dies kann man allerdings ohne genaue bodenkundliche Abklärungen weder beweisen noch widerlegen. Die von Furrer (1969) analysierten Erdstreifenproben aus Ost-Spitzbergen ergeben nur einen verschwindend kleinen Tonanteil. Die Granulogramme von Erdstreifenböden aus unseren Alpen zeigen im wesentlichen dasselbe Bild wie Figur 3, wobei aber der Anteil der Tonfraktion nur ganz gering ausgebildet ist (also wie in OstSpitzbergen). Die Korngrößenverhältnisse am Kilimandjaro sind nun grundsätzlich anders (Fig. 4). Bei allen Proben (in $4 \mathrm{~cm}$ Bodentiefe oft schon anstehender Fels) tritt eine klare Dominanz der Korngrößen 0,2-2 mm zutage, während der Anteil der kleineren Fraktionen unbedeutend ausfällt. Der minime Tongehalt läßt sich dadurch erklären, daß bei häufigen Niederschlägen in den Tropen und beim Fehlen eines Wasserstauers (durchlässiges Vulkangestein, kein Permafrost) das Feinmaterial des Bodens weggeschwemmt wird.

\section{Vergleichende Betrachtung}

Das auffallendste Merkmal beim Vergleich der Kleinformen aus dem arktischen, gemäßigten und tropischen Klimabereich ist der Größenunterschied. Alle Untersuchungen haben bestätigt, daß die Erdknospen und Erdstreifen am Kilimandjaro höchstens halb so groß sind wie die Formen der beiden anderen Arbeitsgebiete. Die alpinen Bodenmuster

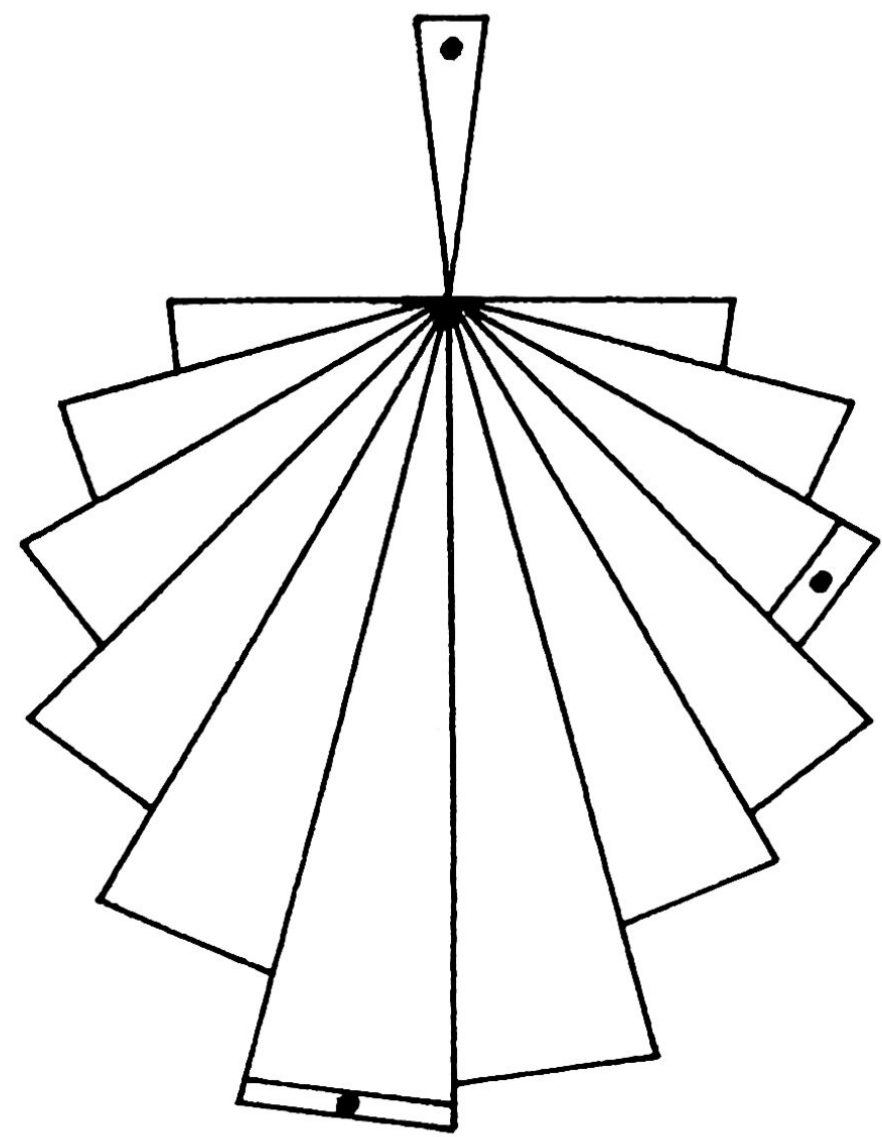

Figur 2. Sammelsitugramm von Erdstreifen in Mittelbünden, gezeichnet aus den Ergebnissen von 6 Messungen $\left(6^{\circ} \mathrm{SW}, 8^{\circ} \mathrm{S}\right.$ nur, $12^{\circ} \mathrm{S}, 17^{\circ} \mathrm{W}, 20^{\circ} \mathrm{W}$, $20^{\circ} \mathrm{S} ; 2440-2810 \mathrm{~m}$ ü. M.)

sind nur geringfügig größer als die arktischen, wobei aus Spitzbergen bloß spärliche Beobachtungen vorliegen.

Aus dem situmetrischen Befund werden keine Unterschiede ersichtlich, wohl aber aus der Granulometrie. Die Korngrößenverteilung am Kilimandjaro ist am eigenwilligsten, aber gut erklärbar. Die Alpen zeigen angenähert eine Gleichverteilung - mit einer zunehmenden Tendenz zu den größeren Fraktionen. Auch hier fehlt der Tonanteil, weil ein dichter Felsuntergrund und der Permafrost als Wasserstauer ebenfalls fehlen. Die Korngrößen aus West-Spitzbergen sind aus den erwähnten Gründen gekennzeichnet durch den relativ hohen Tongehalt.

Trotz all der Unterschiede bleiben die formtypischen Merkmale der Erdknospen und Erdstreifen durchgehend erhalten. Es sind dies:

Feinerde aufgewölbt, mit Blasen im Innern; Grobschutt in flachen Rinnen, ohne Riß;

Rahmenauskleidung; schwebende Formen (Profil); bei $2-6^{\circ}$ Hangneigung Übergänge (längliche Knospen); 


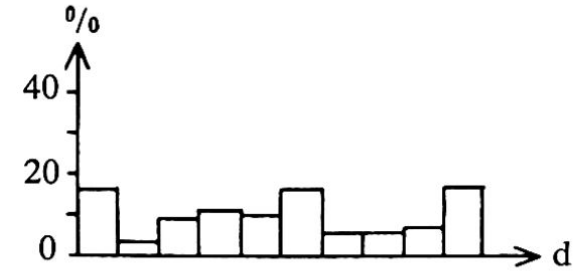

Schuttstreifen, Oberfläche

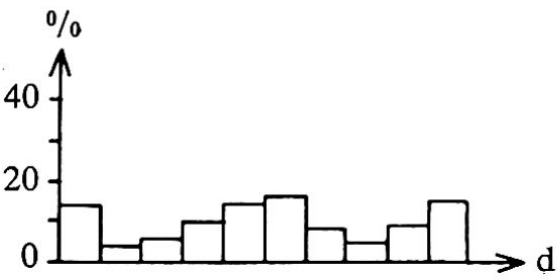

Schuttstreifen, $10 \mathrm{~cm}$ Tiefe

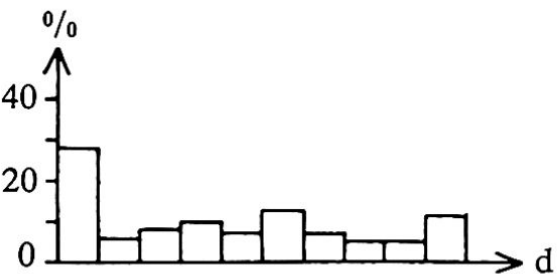

Schuttstreifen, $20 \mathrm{~cm}$ Tiefe
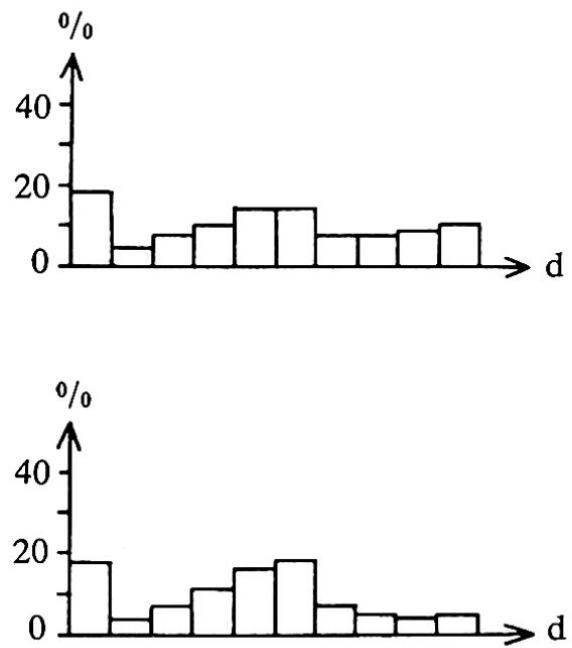

Feinerdestreifen, Oberfläche

Feinerdestreifen, $10 \mathrm{~cm}$ Tiefe

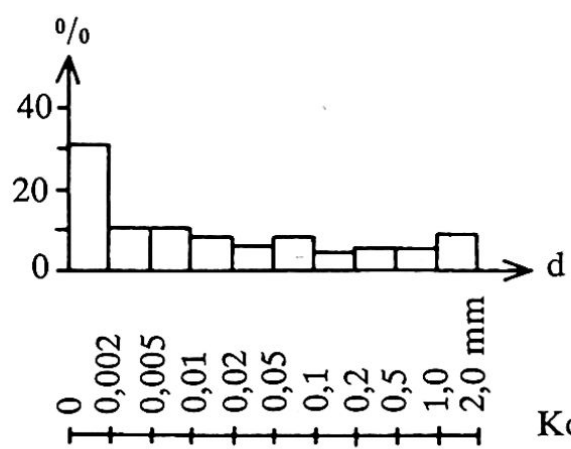

Feinerdestreifen, $20 \mathrm{~cm}$ Tiefe

Korndurchmesser

Figur 3. Granulogramme eines Erdstreifenbodens auf West-Spitzbergen. Hotellneset, $130 \mathrm{~m}$ ü. M., $25^{\circ} \mathrm{E}$
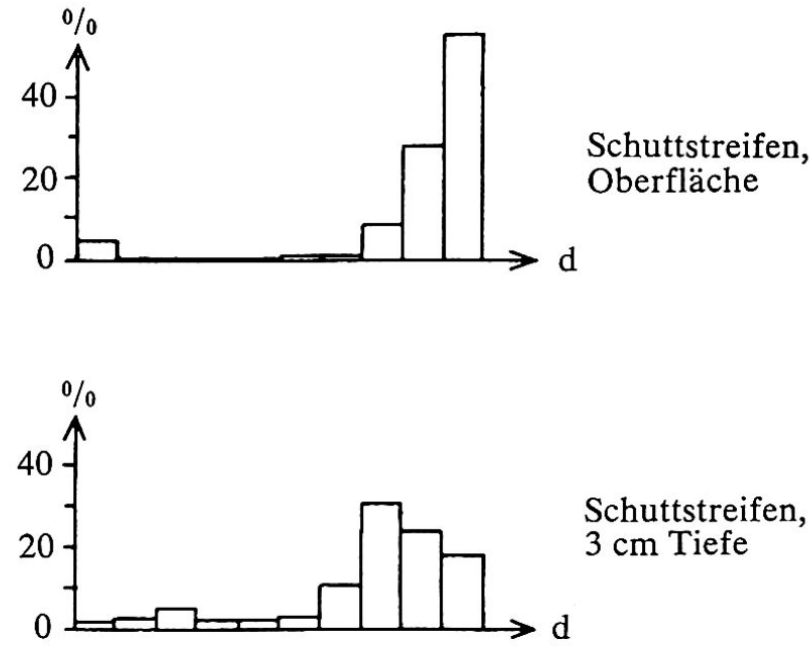

Schuttstreifen, $3 \mathrm{~cm}$ Tiefe

Feinerdestreifen, Oberfläche

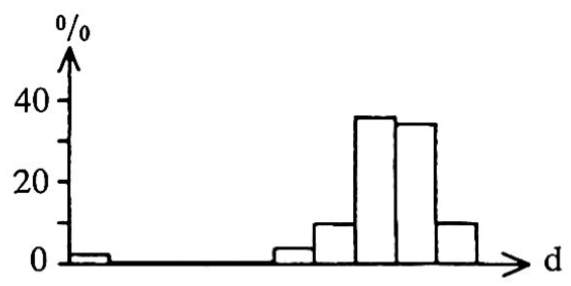

Feinerdestreifen, $3 \mathrm{~cm}$ Tiefe
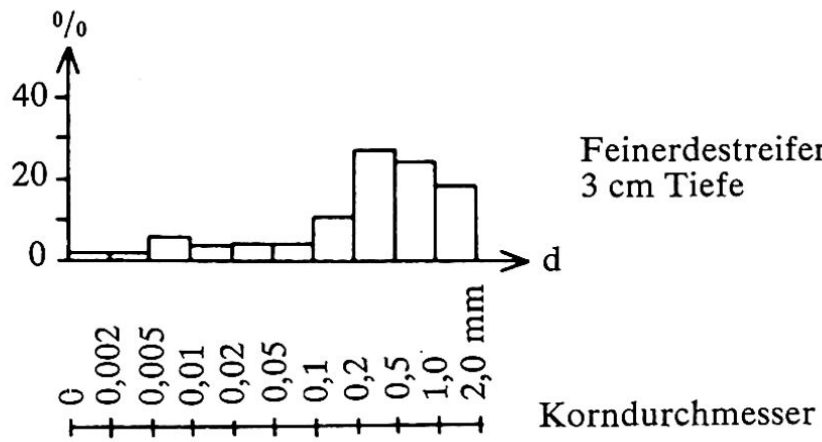

Figur 4. Granulogramme eines Erdstreifenbodens am Kilimandjaro. Sattel, $4330 \mathrm{~m}$ ü. M., $4^{\circ} \mathrm{S}$

\section{Stromlinienverlauf der Erdstreifen um Hindernisse \\ (auf West-Spitzbergen nicht beobachtet).}

Die Entstehung der Erdstreifen scheint wie jene der Erdknospen gesichert. Die Kammeissolifluktion dürfte dabei eine nicht zu unterschätzende Rolle spielen. Im Bündner Untersuchungsgebiet habe ich einige direkte Kammeisbeobachtungen in Erdstreifenfeldern machen können, am Kilimandjaro noch häufigere und schönere. Dort haben Messungen ergeben, daß ein Feinerdestreifen in einer Nacht durch Kammeisnadeln um bis zu $7 \mathrm{~cm}$ gehoben werden kann. Unter den Schuttstreifen finden sich nur ganz winzige Nadeln, aber dieser Schutt wird trotzdem um denselben Betrag gehoben - gewissermaßen passiv durch Eingeklemmtsein zwischen zwei Feinerdestreifen. 


\section{Zusammenfassung}

Zwei Vertreter der Kleinformen von Frostmusterböden - Erdknospen und Erdstreifen - wurden aus drei Untersuchungsgebieten - Spitzbergen, Bündner Alpen, Kilimandjaro - miteinander verglichen. Dabei ergaben sich markante Unterschiede mit Bezug auf die Dimensionen und die Korngrößenverteilung der untersuchten Bodenproben. Weil aber eine ganze Reihe formtypischer Merkmale durchwegs vorhanden war, blieben die Formen nie in Frage gestellt und konnten einem Vergleich standhalten. Die Kleinformen sind keine "tropischen Typen", sondern bilden sich in verschiedenen Frostklimaten der Erde in einer mehr oder weniger deutlichen lokaltypischen Ausprägung.

\section{Résumé}

On a observé deux représentants des formes en miniature de sols structurés dans trois régions différentes au point de vue du climat et de la géographie - le Spitzberg (région arctique), montagnes Grisonnaises, Kilimandjaro (haute montagne tropique). C'étaient des bourgeons de terres (Erdknospen) et des bandes terreuses. Plusieurs marques typiques restaient les mêmes dans les trois régions, mais il y avait de différences au point de vue de la grandeur des formes et de la répartition de la grandeur des grains de pierre. Malgré tout, les formes en miniature ne sont pas de types tropiques - comme on le croyait auparavant; on les voit dans plusieurs climats de gel avec de marques locales.

\section{Literatur}

Bachmann F.: Fossile Strukturböden und Eiskeile auf jungpleistozänen Schotterflächen im nordostschweizerischen Mittelland. Diss. Univ. Zürich, 1966.

Fitze P.: Untersuchungen von Solifluktionserscheinungen im Alpenquerprofil zwischen Säntis und Lago di Como. Diss. Univ. Zürich, 1969.

Flückiger O.: Schuttstrukturen am Kilimandjaro. Petermanns Geogr. Mitt. 11/12, 1934.

Freund R.: Solifluktionsformen am Parpaner Rothorn. Diplomarbeit Univ. Zürich, 1968.

Furrer G.: Solifluktionsformen im Schweizerischen Nationalpark. Diss. Univ. Zürich, 1954.

- Die Strukturbodenformen der Alpen. Geogr. Helv. 4, 1955.

- Untersuchungen am subnivalen Formenschatz in Spitzbergen und in den Bündner Alpen. Geogr. Helv. 4, 1959.

- Die Höhenlage von subnivalen Bodenformen. Habilitationsschrift Univ. Zürich, 1965.

- Vergleichende Beobachtungen am subnivalen Formenschatz in Ost-Spitzbergen und in den Schweizer Alpen. Ergebnisse der Stauferland-Expedition 1959/60, Heft 9, Wiesbaden, 1969.

Kelletat D.: Verbreitung und Vergesellschaftung rezenter Periglazialerscheinungen im Apennin. Göttinger Geogr. Abh. 48, 1969.

- Rezente Periglazialerscheinungen im schottischen Hochland. Göttinger Geogr. Abh. 51, 1970. Mohaupt W.: Beobachtungen über Bodenversetzungen und Kammeisbildungen aus dem Stubai und dem Grödener Tal. Diss. Hamburg, 1932.

Troll C.: Strukturböden, Solifluktion und Frostklimate der Erde. Geol. Rundschau 34, 7/8, 1944. 